\title{
Acceptance Double Sampling Plan with Fuzzy Parameter
}

\author{
Bahram Sadeghpour-Gildeh" ${ }^{1}$, Gholamhossein Yari $^{2}$, Ezzatallah Baloui Jamkhaneh, ${ }^{3, *}$ \\ ${ }^{1}$ Department of Statistics, Faculty of Basic Science, University of Mazandaran Bablosar, Iran \\ Sadeghpour@umz.ac.ir \\ ${ }^{2}$ Iran University of Science and Technology, Tehran, Iran, yari @iust. ac. ir \\ ${ }^{3}$ Department of Statistics, Science and Research Branch, Islamic Azad University, Tehran, Iran. \\ e_baloui2008@yahoo.com.
}

\begin{abstract}
In this present paper we have argued the acceptance double sampling plan when the fraction of defective items is a fuzzy number. we have shown that the operating characteristic (oc) curves of the plan is like a band having a high and low bounds whose width depends on the ambiguity proportion parameter in the lot when that sample size and acceptance numbers is fixed. Finally we completed discuss opinion by a numerical example.
\end{abstract}

Keywords: Statistical quality control, acceptance double sampling, fuzzy number.

\section{Introduction}

Sampling for acceptance or rejecting a lot is an important field in statistical quality control. Acceptance double sampling plan is one of the sampling methods for acceptance or rejection which is along with classical attribute quality characteristic.

In different acceptance sampling plans the fraction of defective items, is considered as a precise value, but sometimes we are not able to obtain exact numerical value, and there also

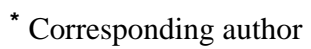

exist some uncertainty in the value of $p$ obtained from experiment, personal judgment or estimation. However the quality characteristic in a lot is not often exact and certain. The theory of fuzzy sets in widely used in solving problems in which parameter or quantities can't be expressed precisely. The theory is a powerful and well-known tool to formulate and analyze the uncertainty resulting from ambiguity and personal judgment. In dealing with the above problem we tried to restore the uncertainty existing in the problem by defining the imprecise parameter as a fuzzy number, and achieve a result with a higher certainty. With this definition, the number of defective items in the sample has a fuzzy binomial probability distribution. Classical acceptance sampling plans have been studied by many researchers. They are thoroughly elaborated by Schilling (1982). Single sampling by attributes with relaxed requirements were discussed by Ohta and Ichihashi (1988) kanagawa and Ohta (1990), Tamaki, Kanagawa and Ohta (1991),and Grzegorzewski(1998,2001b). Grzegrozewski (2000b,2002) also considered sampling plan by variables 
with fuzzy requirements. Sampling plan by attributes for vague data were considered by Hrniewicz (1992, 1994).

We provide some definition and preliminaries of fuzzy sets theory and fuzzy probability in section 2 . In section 3 the fuzzy probability of acceptance of the lot, was considered broadly, and its values in special case was computed. In section 4, we deal with oc band of such a plan, with a example. Finally fuzzy average sample number is introduced in section 5 .

\section{Preliminaries}

Let $X=\left\{x_{1}, \ldots, x_{n}\right\}$ be a finite set $P$ be probability function defined on all subsets of $\mathrm{X}$ with $P\left(\left\{x_{i}\right\}\right)=k_{i}, 1 \leq i \leq n, 0<k_{i}<1$ all $\mathrm{i}$ and $\sum_{i=1}^{n} k_{i}=1 . \mathrm{X}$ together with $P$ is a discrete (finite) probability function. If A be subset of $X$, we have

$$
P(A)=\sum_{x_{i} \in A} P\left(\left\{x_{i}\right\}\right) .
$$

In practice all the $k_{i}$ value must be known exactly. Many times these values are estimated, or they are provided by experts. We now assume that some of these $k_{i}$ values are uncertain and we will model this uncertainty using fuzzy number.

Definition1: the fuzzy subset $\tilde{N}$ of real line IR, with the membership function $\mu_{N}: I R \rightarrow[0,1]$ is a fuzzy number if and only if (a) $\tilde{N}$ is normal (b) $\tilde{N}$ is fuzzy convex (c) $\mu_{N}$ is upper semi continuous (d) supp ( $\tilde{N}$ ) is bounded.

Definition2: A triangular fuzzy number $\tilde{N}$ is fuzzy number that membership function defined by three number $a_{1}<a_{2}<a_{3}$ where the base of the triangle is the interval $\left[a_{1}, a_{3}\right]$ and vertex is at $\mathrm{x}=\mathrm{a}_{2}$.

Definition3: Due to the uncertainty in the $k_{i}$ 's values

we substitute $\tilde{k}_{i}$, a fuzzy number, for each $k_{i}$ and assume that $0<\tilde{k}_{i}<1$ all i. Then $\mathrm{X}$ together with the $\tilde{k}_{i}$ value is a discrete fuzzy probability distribution. We write $\tilde{P}$ for fuzzy $P$ and we have $\widetilde{P}\left(\left\{x_{i}\right\}\right)=\tilde{k}_{i}$. Let $A=\left\{x_{1}, \ldots, x_{l}\right\}$ be subset of $X$. Then define:

$$
\widetilde{P}(A)[\alpha]=\left\{\sum_{i=1}^{l} k_{i} \mid s\right\}
$$

For $0 \leq \alpha \leq 1$, where stands for the statement “ $k_{i} \in \tilde{k_{i}}[\alpha], \underset{i \leq n}{=}, \sum_{i=1}^{n} k_{i}=1$ ” this is our restricted fuzzy arithmetic. The fuzzy mean is defined by its $\alpha$ - cuts:

$$
\tilde{\mu}[\alpha]=\left\{\sum_{i=1}^{k} x_{i} k_{i} \mid s\right\}
$$

where, $\mathrm{S}$ is as before.

Definition4: In $\mathrm{m}$ independent Bernolli experiment let us assume that $p$, probability of a "success" in each experiment is not known precisely and needs to be estimated, or obtained from expert opinion.

So that $p$ value is uncertain and we substitute $\tilde{p}$ for $\mathrm{p}$ and $\tilde{q}$ for $q$ so that there is a $p \in p[1]$ and a $q \in q[1]$ with $p+q=1$.

Now let $\tilde{P}(r)$ be the fuzzy probability of $r$ successes in $m$ independent trials of the experiment. Under our restricted fuzzy algebra we obtain

$$
\tilde{P}(r)[\alpha]=\left\{C_{m}^{r} p^{r} q^{m-r} \mid s\right\}
$$


For $0 \leqq \alpha \leqq 1$, where now $\mathrm{S}$ is the statement,

“ $p \in \tilde{p}[\alpha], q \in \tilde{q}[\alpha], p+q=1$ ”.

If $\widetilde{P}(r)[\alpha]=\left[P_{r_{1}}(\alpha), P_{r_{2}}(\alpha)\right]$ then

$P_{r_{1}}(\alpha)=\min \left\{C_{m}^{r} p^{r} q^{m-r} \mid s\right\}$ and

$P_{r_{2}}(\alpha)=\max \left\{C_{m}^{r} p^{r} q^{m-r} \mid s\right\}$ and if

$\widetilde{P}[a, b]$ be the fuzzy probability of $x$ successes so that fuzzy $\mathrm{a} \leqq x \leqq \mathrm{~b}$, then

$\widetilde{P}([a, b])[\alpha]=\left\{\sum_{x=a}^{b} C_{m}^{x} p^{x} q^{m-x} \mid s\right\}(4)$

if $\tilde{P}([a, b])[\alpha]=\left[P_{1}([a, b])[\alpha], P_{2}([a, b])[\alpha]\right]$ then:

$P_{1}([a, b])[\alpha]=\min \left\{\sum_{x=a}^{b} C_{m}^{x} p^{x} q^{m-x} \mid s\right\}$

and

$\left.P_{2}([a, b])[\alpha]=\max \left\{\sum_{x=a}^{b} C_{m}^{x} p^{x} q^{m-x} \mid s\right\}\right\}$

Where S is the same with past case.

Example1. Suppose that $\mathrm{m}=3$ and $p$ and $q$ are uncertain we use $\tilde{p}=(0.3,0.4,0.5)$ for $p$ and

$\tilde{q}=(0.5,0.6,0.7)$ for $q$. Now we will calculate the fuzzy probabilities $\widetilde{P}(1)$ and $\tilde{P}(A)$ that $\mathrm{A}=\{0,1\}$. If $p \in \tilde{p}[\alpha]$ then $q=1-p \in \tilde{q}[\alpha]$.

Equations of definition 4 become:

$P_{r_{1}}(\alpha)=\min \left\{3 p(1-p)^{2} \mid s\right\}$ and

$P_{r_{2}}(\alpha)=\max \left\{3 p(1-p)^{2} \mid s\right\}$ since

$\frac{\partial\left(3 p(1-p)^{2}\right)}{\partial p}<0$ on $\tilde{p}[0]$

obtain:
$\widetilde{P}(1)[\alpha]=\left[3 p_{2}(\alpha)\left(1-p_{2}(\alpha)\right)^{2}\right.$,

$\left.3 p_{1}(\alpha)\left(1-p_{1}(\alpha)\right)^{2}\right]$

where

$\tilde{p}[\alpha]=\left[p_{1}(\alpha), p_{2}(\alpha)\right]=[0.3+0.1 \alpha, 0.5-0.1 \alpha]$

under $\alpha=0$ we gain

$\widetilde{P}(1)[0]=[0.375,0.441]$ and

$P(A)[\alpha]=\left[P_{A_{1}}(\alpha), P_{A_{2}}(\alpha)\right]$ that

$P_{A_{1}}(\alpha)=\min \left\{(1-p)^{3}+3 p(1-p)^{2} \mid s\right\}$

and

$P_{A_{2}}(\alpha)=\max \left\{(1-p)^{3}+3 p(1-p)^{2} \mid s\right\}$

since

$\frac{\partial\left((1-p)^{3}+3 p(1-p)^{2}\right)}{\partial p}<0$ on $\tilde{p}[0]$

we get

$\widetilde{P}(A)[\alpha]=\left[\left(1-p_{2}(\alpha)\right)^{3}+3 p_{2}(\alpha)\left(1-P_{2}(\alpha)\right)^{2}\right.$, $\left.\left(1-p_{1}(\alpha)\right)^{3}+3 p_{1}(\alpha)\left(1-p_{1}(\alpha)\right)^{2}\right]$ unde $\alpha=0$ we will have $\widetilde{P}(A)[\alpha]=[0.5,0.784]$.

\section{Acceptance double sampling plan with fuzzy parameter}

In this section, first we introduce the double sampling plan for classical attributes characteristics. Suppose that we want to inspect a lot with a size of $\mathrm{N}$. First we choose and inspect a random sample of size $n_{1}$, and then the number of nonconforming items $\left(\mathrm{d}_{1}\right)$ will be count down. If the number of observed nonconforming items is less or equal to acceptance number of first sample, that is $c_{1}$, then the lot will be accepted. And if $\mathrm{d}_{1}$ is greater than to acceptance number of two samples, that is $\mathrm{C}_{2}$, then the lot will be rejected, otherwise we choose second random sample of size $\mathrm{n}_{2}$, then the number of nonconforming items $\left(\mathrm{d}_{2}\right)$ will be count down. In this stage from 
the defective items in each two samples, $\left(d_{1}+d_{2}\right)$, we use for resulting about lot.

If $\mathrm{d}_{1}+\mathrm{d}_{2}$ is less or equals to acceptance number of two samples, then the lot will be accepted, otherwise the lot rejected. If the size of lot was very large, the random variables $d_{1}$ and $d_{2}$ have binomial distribution with parameters $\left(n_{1}, p\right)$ and $\left(n_{2}, p\right)$, in which $p$ indicates the fraction of the lot's nonconforming items. So if we represent probability of acceptance on combined samples with $p_{a}$, and also the probability of the lot's acceptance in first and second samples respectively $p_{a}^{I}, p_{a}^{I I}$ then

$$
p_{a}=p^{I}{ }_{a}+p_{a}^{I I}
$$

That $p_{a}^{I}$ indicates the probability of observation of $\mathrm{d}_{1} \leq C_{1}$ defective items in first random sample. thus

$$
p_{a}^{I}=\sum_{d_{1}=0}^{c_{1}} C_{n_{1}}^{d_{1}} p^{d_{1}}(1-p)^{n_{1}-d_{1}}
$$

And $p_{a}^{I I}$ according to the independence of two random variables and their distribution will calculate with this formula:

$$
p_{a}^{I I}=P\left(\mathrm{~d}_{1}+\mathrm{d}_{2} \leq c_{2}, c_{1}<\mathrm{d}_{1}<c_{2}\right)
$$

Suppose that we want to inspect a lot with the size of $\mathrm{N}$, which the proportion of damaged items or the probability of defectiveness of each of its number is not known precisely and is uncertain value. So we represent this parameter with a fuzzy number $\tilde{p}$ which is:

$\tilde{p}=\left(a_{1}, a_{2}, a_{3}\right), p \in \tilde{p}[1], q \in \tilde{q}[1], p+q=1$
A double sampling plan with a fuzzy parameter is defined by the first sample size $\mathrm{n}_{1}$, acceptance number of first sample $\mathrm{c}_{1}$, second sample size $\mathrm{n}_{2}$, and acceptance number of two samples $\mathrm{C}_{2}$.

If the number of observed nonconforming items on first sample $\left(\mathrm{d}_{1}\right)$ is less or equal to acceptance number of first sample, then the lot will be accepted, and if $d_{1}$ is greater than to acceptance number of two sample, then the lot will be rejected, otherwise we choose second random sample of size $n_{2}$, then the number of nonconforming items $\left(d_{2}\right)$ will be count down. In this case from the total defective items in each two sample $\left(\mathrm{d}_{1}+\mathrm{d}_{2}\right)$, we for resulting about lot. If $\mathrm{d}_{1}+\mathrm{d}_{2}$ is less or equal to acceptance number of two samples, then the lot be accepted, otherwise the lot rejected, If the size of lot was very great, the random variables $d_{1}$ and $d_{2}$ have fuzzy binomial probability distribution with parameters $\left(n_{1}, \tilde{p}\right),\left(n_{2}, \tilde{p}\right)$, in which $\tilde{p}$ indicates the fuzzy proportion of the defective items. According to this case if we show the fuzzy probability of the lot's acceptance in combined samples with $\widetilde{P}_{a}$ and also the fuzzy probability of the lot's acceptance in first and second samples respectively $\tilde{p}_{a}^{I}, \tilde{p}_{a}^{I I}$ then

$$
\tilde{p}_{a}=\tilde{p}_{a}^{I}+\tilde{p}_{a}^{I I}
$$

Example2. Suppose that $\tilde{p}=(0.01,0.02,0.03)$ and $\tilde{q}=(0.97,0.98,0.99)$ and $n_{1}=10$, $n_{2}=10, c_{1}=0$, $c_{2}=1$, then fuzzy probability of acceptance of this lot is as follows:

$$
\tilde{p}_{a}^{I}[\alpha]=\tilde{p}_{\mathrm{d} 1}(0)[\alpha]=\left\{(1-p)^{10} \mid s\right\}
$$




$$
\begin{aligned}
& \text { Since } \frac{\partial\left((1-p)^{10}\right)}{\partial p}<0 \text { on } \\
& \tilde{p}[\alpha]=[0.01+0.01 \alpha, 0.03-0.01 \alpha], 0<\alpha<1 \text {, } \\
& \text { weobtain }
\end{aligned}
$$$$
\tilde{p}_{a}^{I}[\alpha]=\left[(0.97+0.01 \alpha)^{10},(0.99-0.01 \alpha)^{10}\right]
$$$$
\text { under } \alpha=0
$$$$
\text { we gain } \tilde{p}_{a}^{I}[0]=[0.7374,0.9044]
$$$$
\tilde{p}_{a}^{I I}[\alpha]=\widetilde{P}\left(\mathrm{~d}_{1}=1, \mathrm{~d}_{1}+\mathrm{d}_{2}<1\right)[\alpha]=
$$$$
\left\{10 p(1-p)^{14} \mid s\right\} \text { since }
$$$$
\frac{\partial\left(10 p(1-p)^{14}\right)}{\partial p}>0 \text { on } \tilde{p}[\alpha] \text { therefor }
$$$$
\tilde{p}_{a}^{I I}[\alpha]=\left[10(0.01+0.01 \alpha)(0.99-0.01 \alpha)^{14}\right. \text {, }
$$$$
\left.10(0.03-0.01 \alpha)(0.97+0.01 \alpha)^{14}\right]
$$

under $\alpha=0$ we obtain

$\tilde{p}_{a}^{I I}[0]=[0.0869,0.1958]$

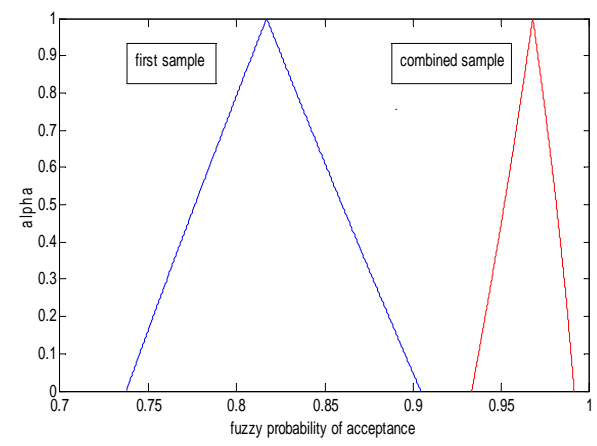

Fig1: fuzzy probability of acceptance with

$$
\widetilde{p}=(0.01,0.02,0.03) \text {, }
$$

Finally wehave $\tilde{p}_{a}[\alpha]=\tilde{p}_{a}^{I}[\alpha]+\tilde{p}_{a}^{I I}[\alpha]$

$$
=\left[(0.97+0.01 \alpha)^{10}+10(0.03-0.01 \alpha)\right.
$$

$(0.97+0.01 \alpha)^{14},(0.99-0.01 \alpha)^{10}$

$\left.+10(0.01+0.01 \alpha)(0.99-0.01 \alpha)^{14}\right]$

then $\tilde{p}_{a}[0]=[0.9332,0.9913]$

\section{4. oc -band with fuzzy parameter}

In assessing a sampling plan, one of the important criteria is its operating characteristic (oc) curve. This curve indicates the probability of a lot acceptance in terms of different values of the fraction of defective items. A double sampling plan has a main oc curve that show the probability of acceptance on combined samples. This plan also has another oc curve that indicates the probability of acceptance base on the first sample.

In double sampling plan with fuzzy parameter, main oc curve is as a band with up and down bound. This band indicates the fuzzy probability of lot's acceptance in terms of different values of the fuzzy proportion of nonconforming items.

The uncertainty degree of a proportion parameter is one of the factors that bandwidth depends on that. The less and uncertainty value results in less bandwidth, and if proportion parameter gets a crisp value, lower and upper bounds will become equal, which that oc curve is in classic state. Knowing the uncertainty degree of proportion parameter (given $\mathrm{a}_{1}, \mathrm{a}_{2}, \mathrm{a}_{3}$ ) and variation of its position on horizontal axis, we have different fuzzy number $(\tilde{p})$ and hence we will have different proportion (p) which the oc bands are plotted in terms of it. To achieve this aim we consider the structure of $\tilde{p}$ as follows:

$$
\tilde{p}=\left(k, \quad \mathrm{a}_{2}+k \quad, \mathrm{a}_{3}+k\right),
$$$$
p \in \tilde{p}[1], q \in \tilde{q}[1], p+q=1
$$

Which with variation of $k$ in the domain of $\left[0,1-a_{3}\right]$, the main oc band, is plotted according to the calculation of follow fuzzy probability:

$$
\begin{aligned}
& \tilde{p}[\alpha]=\left[p_{1}(\alpha), p_{2}(\alpha)\right] \\
& =\left[k+a_{2} \alpha, a_{3}+k-\left(a_{3}-a_{2}\right) \alpha\right]
\end{aligned}
$$


$\tilde{p}_{a}=\tilde{p}_{a}^{I}+\tilde{p}_{a}^{I I}$

$\tilde{p}_{a}^{i}=\tilde{P}_{k}\left(A_{i}\right)[\alpha]=\left[\tilde{P}_{k, A_{i 1}}(\alpha), \tilde{P}_{k, A_{i 2}}(\alpha)\right]$

$, i=I, I I$

$\widetilde{P}_{k, A_{i 1}}(\alpha)=\min \left\{P\left(A_{i 1}\right) \mid s\right\} \quad$ and

$\widetilde{P}_{k, A_{i 2}}(\alpha)=\max \left\{P\left(A_{i 2}\right) \mid s\right\}, i=I, I I$

That the event $A_{i}$ is the event of acceptance of a lot in terms sample ith, and the oc band in term first sample is plotted according to the calculation of follow fuzzy probability:

$\tilde{p}_{a}^{I}=\widetilde{P}_{k}\left(A_{1}\right)[\alpha]=\left[P_{k, A_{11}}(\alpha), P_{k, A_{12}}(\alpha)\right]$

$P_{k, A_{11}}(\alpha)=\min \left\{\sum_{A_{1}} C_{n_{1}}^{d_{1}} p^{d_{1}} q^{n_{1}-d_{1}} \mid s\right\}$ and

$P_{k, A_{12}}(\alpha)=\max \left\{\sum_{A_{1}} C_{n_{1}}^{d_{1}} p^{d_{1}} q^{n_{1}-d_{1}} \mid s\right\}$

\section{Example3.}

suppose taht $n_{1}=10, c_{1}=1$,

$n_{2}=5, c_{2}=2, a_{2}=0.01, a_{3}=0.02$

then we have

$\tilde{p}[\alpha]=(k+0.01 \alpha, 0.02+k-0.01 \alpha), 0<k \ll 0.98$

and if $\alpha=0$ then fuzzy probability of acceptance on first sample is :

$\tilde{p}_{a}^{I}=\tilde{P}_{k}[0,1][0]=\left\{(1-p)^{10}+10 p(1-p)^{9} \mid s\right\}$

$f_{1}(p)=(1-p)^{10}+10 p(1-p)^{10}$

According to that the $f_{1}(p)$ decreasing, then:

$$
\begin{aligned}
& \tilde{p}_{a}^{I}=\left[(0.98-k)^{10}+10(0.02+k)(0.98-k)^{9}\right. \\
& \left.,(1-k)^{10}+10 k(1-k)^{9}\right]
\end{aligned}
$$

and fuzzy probability of acceptance on second sample is :

$$
\begin{aligned}
& \tilde{p}_{a}^{I I}=\widetilde{P}\left(\mathrm{~d}_{1}=2, \mathrm{~d}_{2}=0\right)=\left\{45 p^{2}(1-p)^{13} \mid s\right\} \\
& , f_{2}(p)=45 p^{2}(1-p)^{13}
\end{aligned}
$$

Then with studying $f_{2}(p)$ function, we will have the $\alpha$-cut in the following fashion:

$$
\begin{aligned}
& \left\{\begin{array}{c}
{\left[45 k^{2}(1-k)^{13}, 45(k+0.02)^{2}(0.98-k)^{13}\right]} \\
, 0 \varangle k<\frac{1.7}{15}
\end{array}\right. \\
& {\left[45 k^{2}(1-k)^{13}, 0.1249\right.} \\
& , \frac{1.7}{15} \triangleleft k<0.123544 \\
& \tilde{p}_{a}^{I I}=\left\{\quad \left[45(k+0.02)^{2}(0.98-k)^{13}, 0.1249\right.\right. \\
& , 0.12354 \underset{=}{=}<\frac{2}{15} \\
& {\left[45(k+0.02)^{2}(0.98-k)^{13}, 45 k^{2}(1-k)^{13}\right]} \\
& , \frac{2}{15} \triangleleft k<0.98
\end{aligned}
$$

Figure 2 represents that when the process quality decrease from a very good state to a moderate state, then the oc band will be wider.

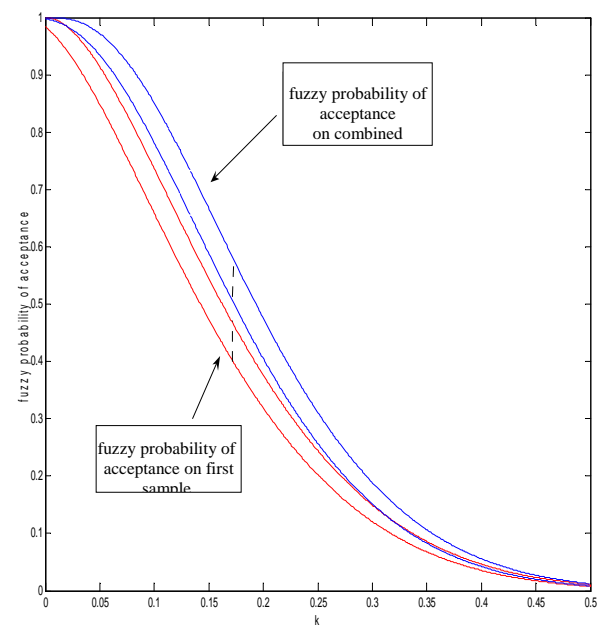

Fig 2: oc band for a double sampling plan with fuzzy parameter of $n_{1}=10 c_{1}=1, n_{2}=5, c_{2}=2$

Using fuzzy arithmetic, and simplification, $\widetilde{p}_{a}$ and table1 will calculate. 
Table1: fuzzy probability of acceptance with $c_{1}=1, n_{1}=10, c_{2}=2, n_{2}=5$

\begin{tabular}{|c|c|c|c|}
\hline$\tilde{p}$ & $\tilde{p}_{a}^{I}$ & $\tilde{p}_{a}^{I I}$ & $\tilde{p}_{a}$ \\
\hline$[0,0.02]$ & {$[0.9838,1]$} & {$[0,0.0138]$} & {$[0.9976,1]$} \\
\hline$[0.01,0.03]$ & {$[0.9655,0.9957]$} & {$[0.003,0.0273]$} & {$[0.9928,0.9987]$} \\
\hline$[0.02,0.04]$ & {$[0.9418,0.9838]$} & {$[0.0138,0.0423]$} & {$[0.9841,0.9976]$} \\
\hline$[0.03,0.05]$ & {$[0.9139,0.9655]$} & {$[0.0273,0.0577]$} & {$[0.9716,0.9928]$} \\
\hline$[0.04,0.06]$ & {$[0.8824,0.9418]$} & {$[0.0423,0.0725]$} & {$[0.9549,0.9841]$} \\
\hline$[0.05,0.07]$ & {$[0.8483,0.9139]$} & {$[0.0577,0.0858]$} & {$[0.9341,0.9716]$} \\
\hline$[0.06,0.08]$ & {$[0.8121,0.8824]$} & {$[0.0725,0.0974]$} & {$[0.9059,0.9549]$} \\
\hline
\end{tabular}

\section{Fuzzy average sample number (FASN)}

The main advantage of double sampling plan is the reduced average sample size, required to arrive to a good decision. In this plan sample number (SN) can be $n_{1}$ or $\mathrm{n}_{1}+\mathrm{n}_{2} \cdot \widetilde{P}_{I}$ is the fuzzy probability of drawing a " first " sample only, which occurs when arriving at a decision at the first sample (with fuzzy probability $\widetilde{P}\left(\mathrm{~d}_{1} \leq c_{1}\right.$ or $\left.\left.\mathrm{d}_{1}>c_{2}\right)\right)$. the fuzzy probability $\widetilde{P}_{I I}$ of having draw a second sample, totaling a size of $n_{1}+n_{2}$, occurs when we had an "inconclusive" outcome from the first sample (with fuzzy probability $\left.\tilde{P}\left(c_{1}<\mathrm{d}_{1} \leq c_{2}\right)\right)$.

The FASN is calculated following the definitions fuzzy mean:
$\mathrm{FASN}=\tilde{\mu}_{S N}[\alpha]=\left\{n_{1} p_{I}+\left(n_{1}+n_{2}\right) p_{I I} \mid s\right\}$

Where, as before, $\mathrm{S}$ denotes the statement

“ $p_{i} \in \tilde{p}_{i}[\alpha], i=I, I I, p_{I}+p_{I I}=1$ ”.

Hence we get

$\mathrm{FASN}=\left\{n_{1}+n_{2} p_{I I} \mid s\right\}$

Example4.

Let that $c_{1}=0, c_{2}=1, n_{1}=10$, $n_{2}=10$ and $a_{2}=0.01, a_{3}=0.02$ thenFASN is as follows:

$\tilde{p}[\alpha]=[k+0.01 \alpha, k+0.02-0.01 \alpha]$ FASN $=\left\{10+100 \mathrm{p}(1-\mathrm{p})^{9} \mid \mathrm{s}\right\}$ then $\alpha$ cut is:

Table2: FASN in terms of different $\tilde{p}$ and $\alpha$

\begin{tabular}{|c|c|c|c|}
\hline$\alpha \backslash k$ & 0 & 0.01 & 0.02 \\
\hline 0 & {$[10,11.67]$} & {$[10.91,12.28]$} & {$[11.67,12.77]$} \\
\hline 0.25 & {$[10,11.49]$} & {$[11.12,12.14]$} & {$[11.83,12.66]$} \\
\hline 0.5 & {$[10.48,11.3]$} & {$[11.31,11.99]$} & {$[11.99,12.54]$} \\
\hline 0.75 & {$[10.7,11.12]$} & {$[11.49,11.83]$} & {$[12.14,12.41]$} \\
\hline 1 & {$[10.91,10.91]$} & {$[11.67,11.67]$} & {$[12.28,12.28]$} \\
\hline
\end{tabular}




$$
\text { FASN }[\alpha]=\left\{\begin{array}{c}
{\left[\text { FASN }^{*}, \text { FASN }^{* *}\right]} \\
, 0<k<0.08 \\
{\left[\text { FASN }^{*}, 13.874\right]} \\
, 0.08<k<0.09029 \\
{\left[\mathrm{FASN}^{* *}, 13.874\right]} \\
, 0.09029<k<0.1 \\
{\left[\mathrm{FASN}^{* *}, \mathrm{FASN}^{*}\right]} \\
, 0.1<k<0.98
\end{array}\right.
$$

where

$$
\begin{aligned}
& \text { FASN }=10+100 k+0.01 \alpha)(1-k-0.01 \alpha)^{9} \\
& \text { andFASN }{ }^{* *}=(10+10 \propto k+0.02-0.01 \alpha) \\
& \left.(0.98-k-0.01 \alpha)^{9}\right)
\end{aligned}
$$

Figure 3 shows FASN band for double sampling plan. If the process quality is very good or very bad, then the lot will be accepted or rejected respectively by the first sample. As a result, the size of the plan sample will be of lower values, and if the process quality is moderate, then in most case, as to the decision on accepting or rejecting the lot, a second sample should be selected which will lead to an increase in the size of the plan sample.

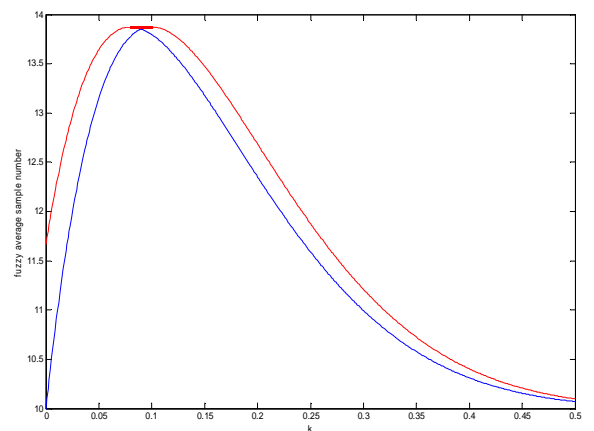

Fig 3: fuzzy average sample number for a double sampling plan with parameter fuzzy

$$
\text { of , } c_{1}=0, c_{2}=1, \quad n_{1}=n_{2}=10
$$

\section{Conclusion}

In the present paper we have proposed a method for designing acceptance double sampling plans with fuzzy quality characteristic. These plans are well defined since if the fraction of defective items is crisp they reduce to classical plans. As it was shown that oc curves of the plan is like a band having a high and low bounds. In this plan if the process quality is very good or very bad, then the fuzzy average sample number will be of lower values.

\section{References:}

[1] J. J. Buckley (2003) fuzzy probability: new approach and application, physica-velage, Hidelberg ,Germany.

[2] J.J. Buckley (2006) fuzzy probability and statistics, springer-verlage Berlin Hidelberg.

[3] D. Dubis , H. Prade (1978)

Operations of fuzzy number, Int. J. syst.

[4] P. Grzegorzewski(1988) A soft design of acceptance sampling by attributes, in: proceedings of the VIth international workshop on intelligent statistical quality control Wurzburg, September 14-16 , PP.29-38.

[5] P. Grzegorzewski (2001 b) Acceptance sampling plans by attributes with fuzzy risks and quality levels, in: Frontiers in frontiers in statistical quality control. Vol. 6, Eds. Wilrich P. Th. Lenz H. J. Springer, Heidelberg, PP. 36-46.

[6] P. Grzegorzewski (2002) A soft design of acceptance sampling plans by variables, in: technologies for 
contrueting intelligent systems, Eds, speringer, vol. 2. pp. 275-286.

[7] O. Hryniewisz (1992) statistical acceptance sampling with uncertain information from a sample and fuzzy quality criteria working paper of $S R I$ $P A S$, Warsow, (in polish).

[8] A. Kanagawa,H. Ohta (1990), A design for single sampling attribute plan based on fuzzy set theory, fuzzy sets and systems, 37. 173-181.

[9] D. C. Montgomery (1991), introduction to statistical quality control, wiley New york.

[10] H. Ohta ,H. Ichihashi (1998), Determination of single-sampling attribute plans based on membership function, Int. J. Prod, Res 26, 14771485.

[11] J. L. Romeu, Understanding binomial sequential testing, Rac start, Volume 12, Number 2.

[12] E.G. Schiling (1982), acceptance sampling quality control, Dekker, New york.

[13] F. Tamaki , A. Kanagawa, Ohta H. (1991), A fuzzy design of sampling inspection plans by attributes, Japanese journal of fuzzy theory and systems, 3 , 315-327. 